\title{
Population level impact of vouchers on access in Uganda
}

\author{
Benjamin Bellows \\ Population Council \\ Francis Obare \\ Population Council \\ Reproductive Health Vouchers Evaluation Team
}

Follow this and additional works at: https://knowledgecommons.popcouncil.org/departments_sbsr-rh

Part of the Demography, Population, and Ecology Commons, Health Policy Commons, and the International Public Health Commons

How does access to this work benefit you? Let us know!

\section{Recommended Citation}

Bellows, Benjamin, Francis Obare, and On behalf of the RH Vouchers evaluation team. 2012. "Population level impact of vouchers on access in Uganda," presentation at the Dissemination of Impact Evaluation Findings Workshop, 23 March 2012, Kampala, Uganda. 


\title{
Population level impact of vouchers on access in Uganda
}

\author{
Ben Bellows, PhD \\ Francis Obare, PhD
}

On behalf of the RH Vouchers evaluation team

Dissemination of Impact Evaluation Findings Workshop

March 23, 2012

Kampala, Uganda

(2) Population Council 


\section{Background}

- Use of vouchers are part of interventions aimed at influencing demand for health services

o conditional cash transfers, social health insurance

$\circ$ approaches referred to as output-based aid (OBA)

- Combined with output-based approach and contracting with providers, its ultimate aims are to:

o stimulate demand by increasing purchasing power for service utilization among the poor

- Trigger competition leading to improved service quality

o Increase access to services for individuals who would not have used the service in the absence of the subsidy

(2) Population Council 


\section{Voucher Program Design \& Functions}

\section{Government stewardship \& funding}

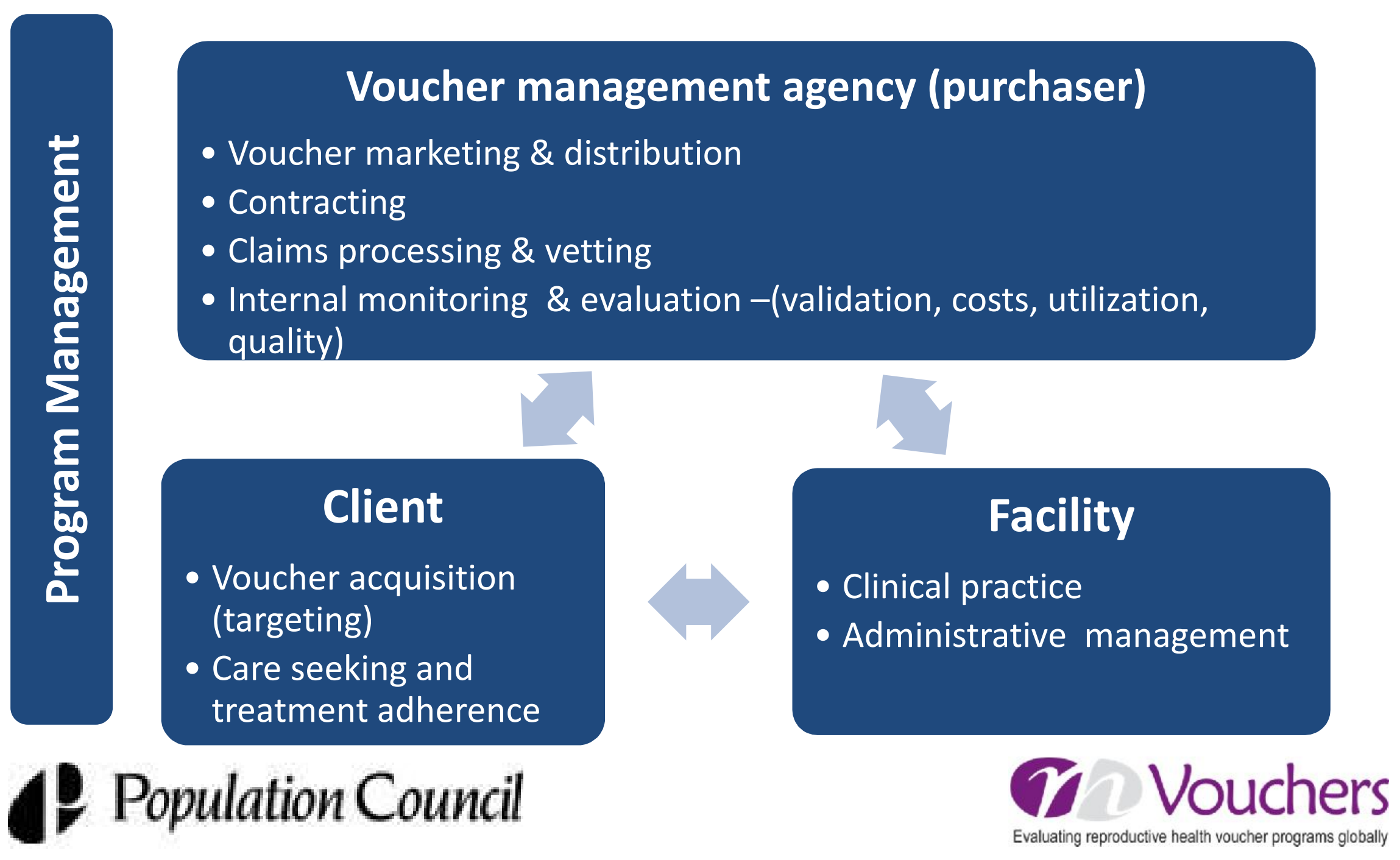




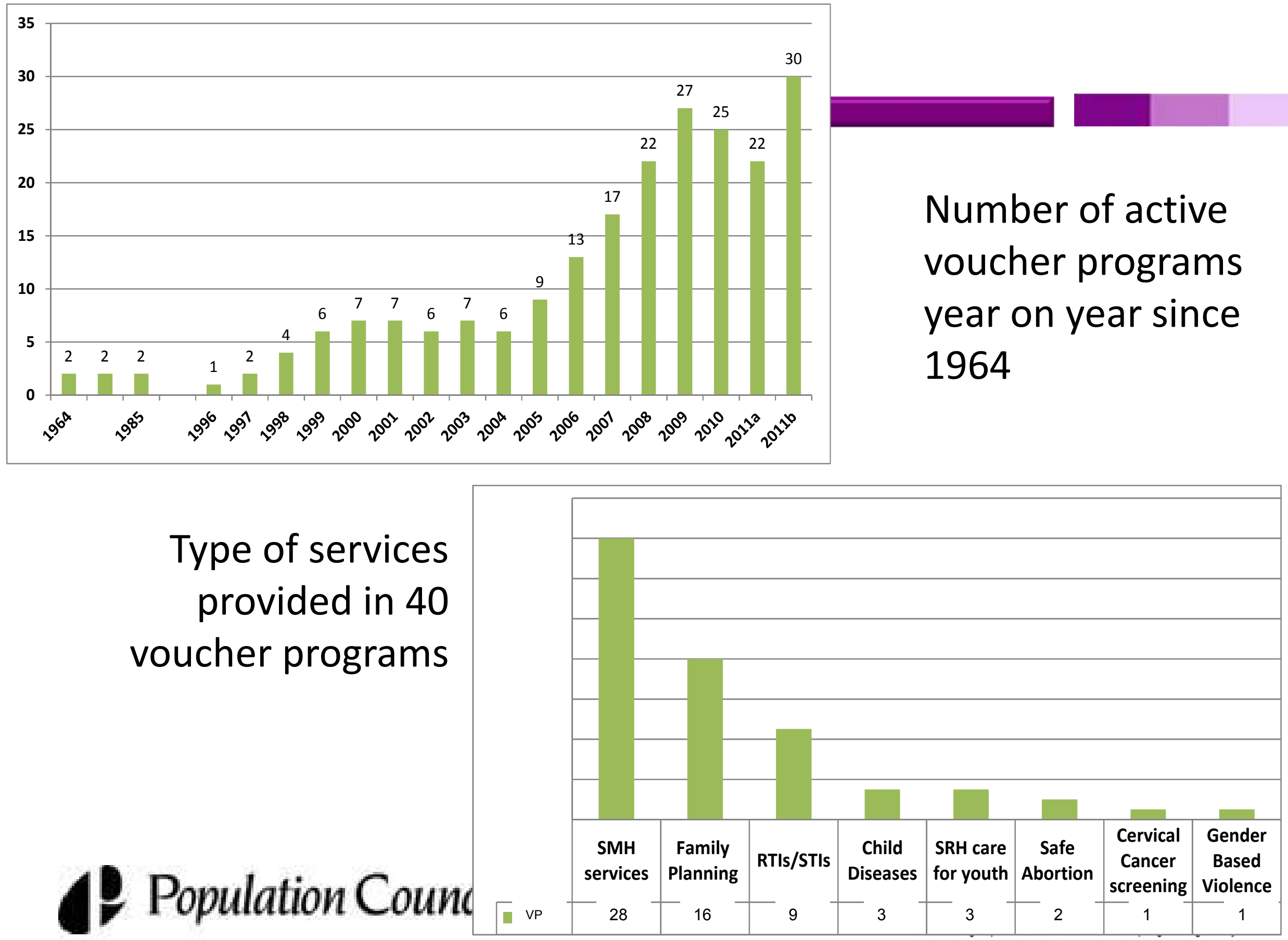




\section{Two voucher systematic reviews}

- Robust evidence that vouchers increase utilization

(13 studies)

- Weak evidence that vouchers can affect health status

(6 studies); however, small changes in the evidence

could change conclusion

- Modest evidence that vouchers effectively target

specific populations for health goods/services

(4 studies)

- Modest evidence that vouchers improve the quality

(3 studies)

- Insufficient evidence to determine efficiency of

vouchers (1 study)

(2) Population Council 


\section{Overview of Uganda RH vouchers program}

- Implemented on behalf of MOH by Marie Stopes Uganda since 2006.

- Phase I: 2006-2008 (KfW STI evaluation)

- Mbarara, Ibanda, Isingiro, Kiruhura

- 17 private facilities saw STI clients

- Phase II: 2008-2011 (GPOBA impact evaluation)

○ 85+ private facilities across western 20+ districts

○ Safe motherhood package (ANC, delivery, PNC), STI treatment

- GPOBA paid $98 \%$ of voucher service delivery cost

- Phase III: 2012-2015

- Family planning services \& safe delivery

- FP: 900 facilities to receive outreach teams; 500 private facilities to be contracted in a voucher franchise

(2) Population Council 


\section{Voucher Distribution and Eligibility}

- Vouchers distributed by Marie Stopes as the Voucher Management Agency (VMA)

- Poverty grading tool used to identify clients (FP \& $\mathrm{SMH})$

o items on household assets, amenities, expenditure, income, health services

- Safe motherhood includes

- ANC up to 4 visits

O delivery and complications

- PNC up to 6 weeks

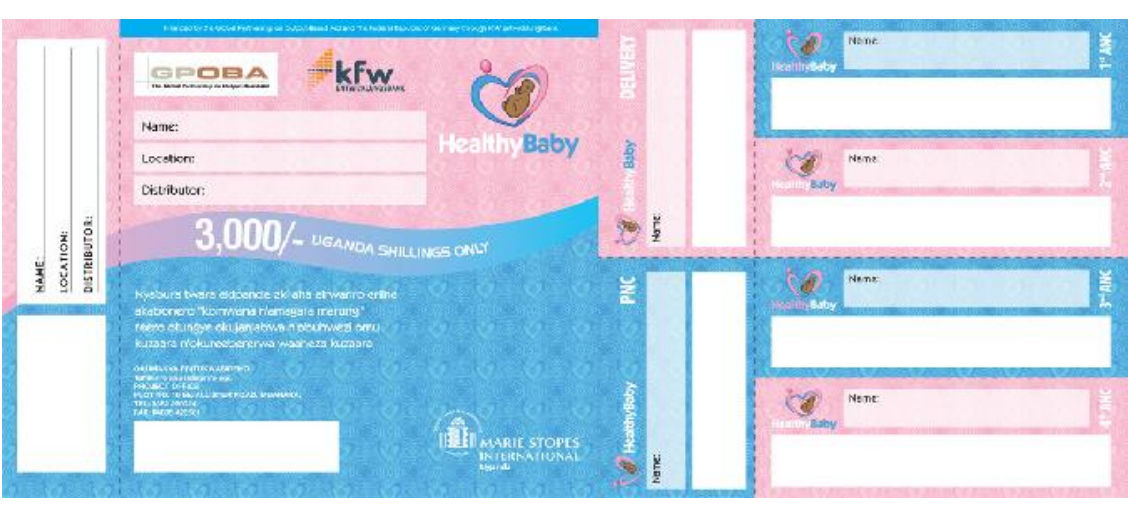

(2) Population Council 


\section{SMH impact evaluation objectives}

1. To assess the effect of the program on improving access to, quality of, and reducing inequities in the use of reproductive health services; and

2. To evaluate the impact of the program on improving reproductive health behaviors and outcomes at the population level. 


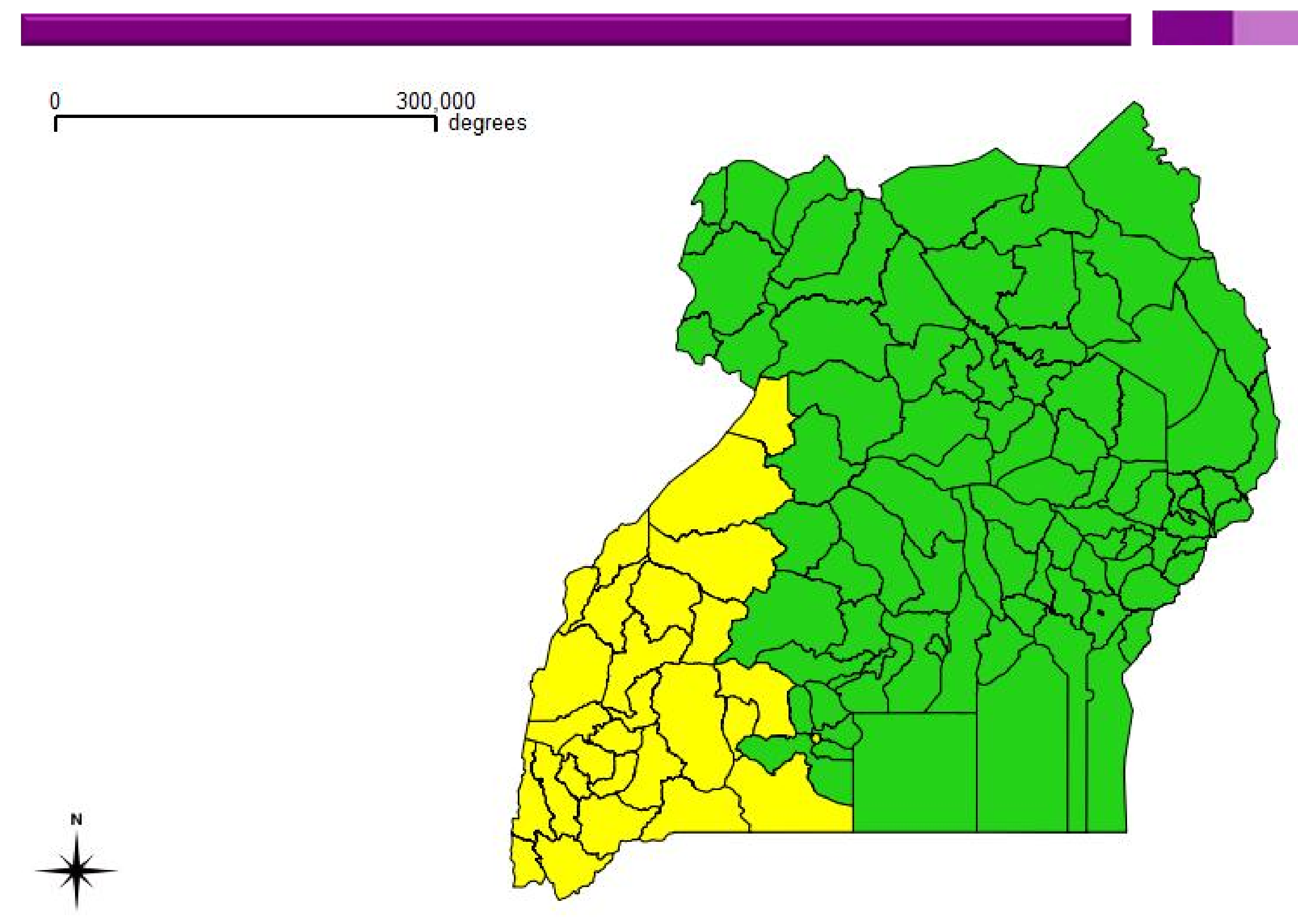




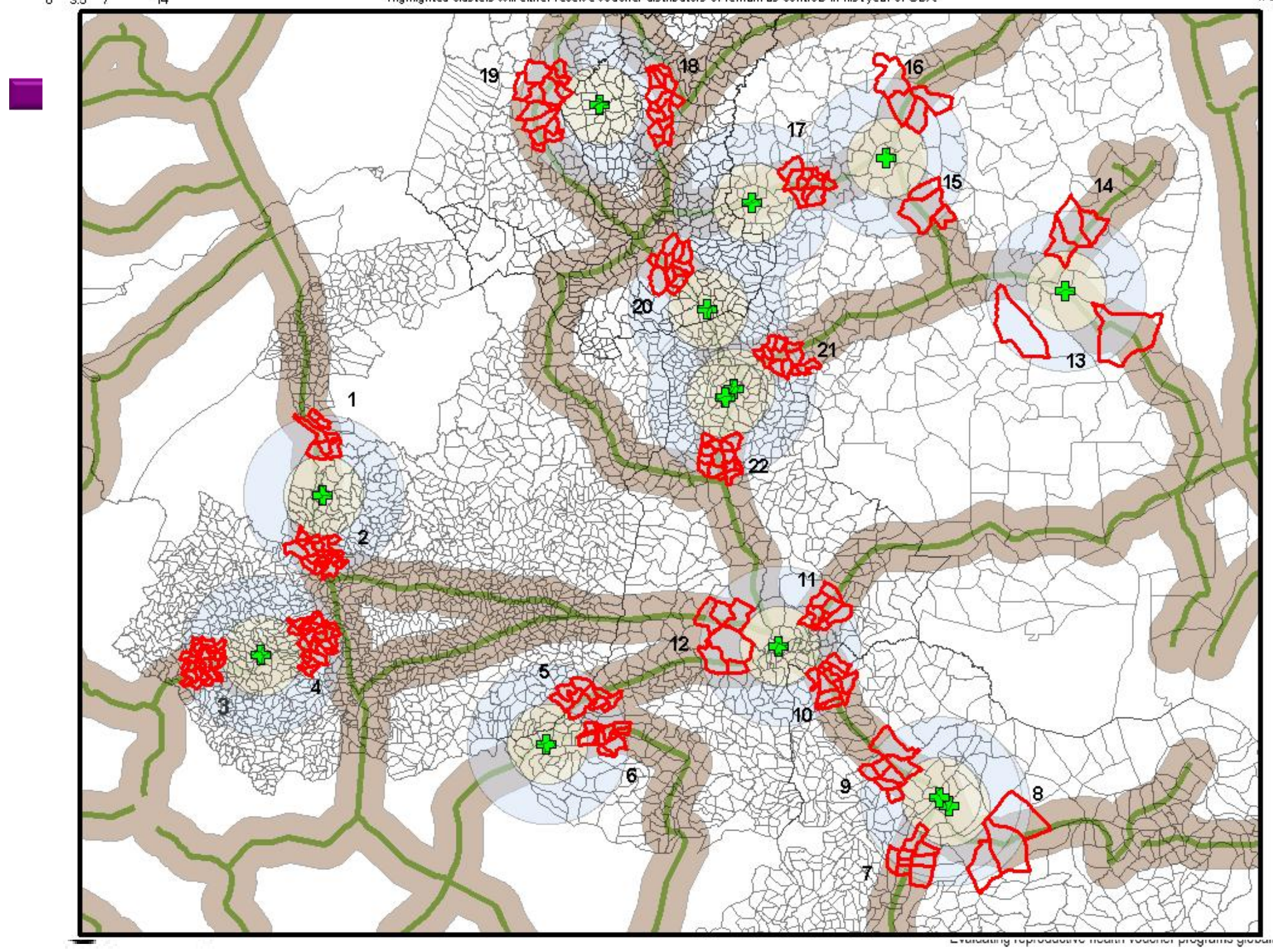




\section{Results chain for SMH voucher}

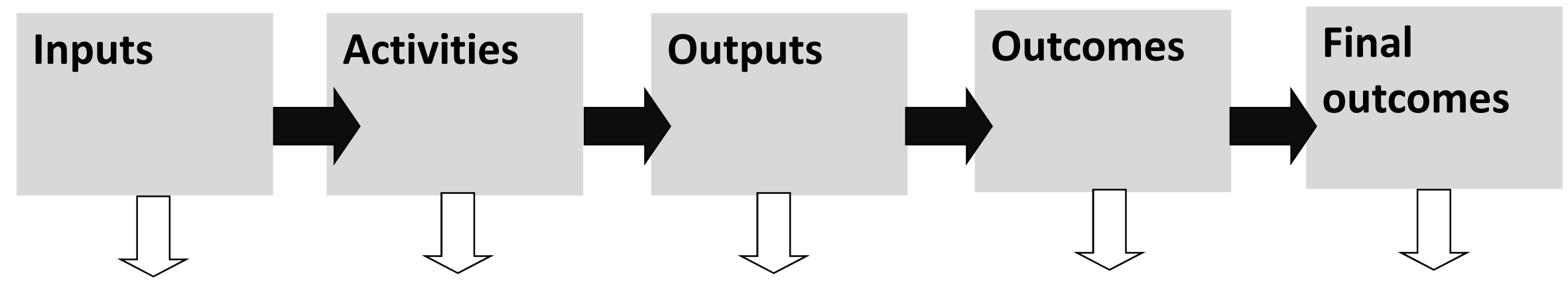

\begin{tabular}{|c|c|c|c|c|}
\hline $\begin{array}{l}\text { Budget for } \\
\text { voucher } \\
\text { service } \\
\text { delivery \& } \\
\text { demand } \\
\text { generation } \\
\text { activities }\end{array}$ & $\begin{array}{l}\text { Contract }+90 \\
\text { private } \\
\text { facilities \& } \\
\text { engage } \\
\text { community- } \\
\text { based } \\
\text { distributors }\end{array}$ & $\begin{array}{l}\text { Sell more than } \\
100,000 \text { safe } \\
\text { motherhood } \\
\text { vouchers }\end{array}$ & $\begin{array}{l}\text { Clients use } \\
\text { voucher to } \\
\text { be seen for } \\
\text { ANC, } \\
\text { delivery and } \\
\text { PNC services }\end{array}$ & $\begin{array}{l}\text { Use of facility } \\
\text { for deliveries } \\
\text { increases; } \\
\text { inequities } \\
\text { decrease; } \\
\text { access } \\
\text { improves }\end{array}$ \\
\hline
\end{tabular}




\section{Impact evaluation design}

\begin{tabular}{|c|c|c|c|c|}
\hline & & 2008 & OBA voucher program & 2010/11 \\
\hline $\begin{array}{c}\text { Voucher } \\
\text { SMH }\end{array}$ & $\begin{array}{c}\text { exposed } \\
\text { villages }\end{array}$ & $X$ & $\longrightarrow$ & X \\
\hline & $\begin{array}{l}\text { Control } \\
\text { villages }\end{array}$ & O & $\longrightarrow$ & 0 \\
\hline
\end{tabular}

Household surveys:

- Baseline (2008): 2,266 women and 177 men in 97 villages

- Endline (2010): 2,313 women and 582 men in 133 villages

(2) Population Council 


\section{Analysis}

- Post hoc treatment assignment

- Analysis 1

- Treatment: voucher clients

- Controls: non-voucher clients

- Analysis 2

- Treatment: Villages with voucher clients

- Controls: Villages no voucher clients

- Difference-in-difference multivariate modeling for tests of association

12 Population Council 


\section{Results 1: Use of voucher by poor*}

Percentage of women who participated in the 2010-2011 survey that had ever used the HealthyBaby voucher by household wealth index

Household wealth index

Poorest quintile

Poorer quintile

Middle quintile

Richer quintile

Richest quintile
Percent

29.3

26.9

16.5

19.4

16.2

21.7
Number of

women

482

442

449

465

475

2,313 


\section{Results 1: Use of any facility for delivery}

\begin{tabular}{|c|c|c|c|c|c|c|}
\hline & \multicolumn{2}{|c|}{ Voucher clients (\%) } & \multicolumn{2}{|c|}{$\begin{array}{c}\text { Non-voucher } \\
\text { clients (\%) }\end{array}$} & \multirow[b]{2}{*}{$\begin{array}{c}\text { Percentage } \\
\text { points }^{a}\end{array}$} & \multirow[b]{2}{*}{$\begin{array}{l}\text { Odds } \\
\text { ratios }^{b}\end{array}$} \\
\hline & $\begin{array}{c}\text { Before } \\
\text { program }\end{array}$ & $\begin{array}{c}\text { After } \\
\text { program }\end{array}$ & $\begin{array}{c}\text { Before } \\
\text { program }\end{array}$ & $\begin{array}{c}\text { After } \\
\text { program }\end{array}$ & & \\
\hline Place of delivery & $(N=175)$ & $(N=434)$ & $(N=708)$ & $(\mathrm{N}=1184)$ & & \\
\hline Home & $30 \%$ & $17 \%$ & $38 \%$ & $31 \%$ & 6 & $\begin{array}{c}0.6 * \\
{[0.3-0.9]}\end{array}$ \\
\hline Any facility & $70 \%$ & $82 \%$ & $61 \%$ & $69 \%$ & 4 & $\begin{array}{c}1.6 \\
{[0.9-2.8]}\end{array}$ \\
\hline
\end{tabular}

(2) Population Council 


\section{Results 2: Use of private facilities for delivery}

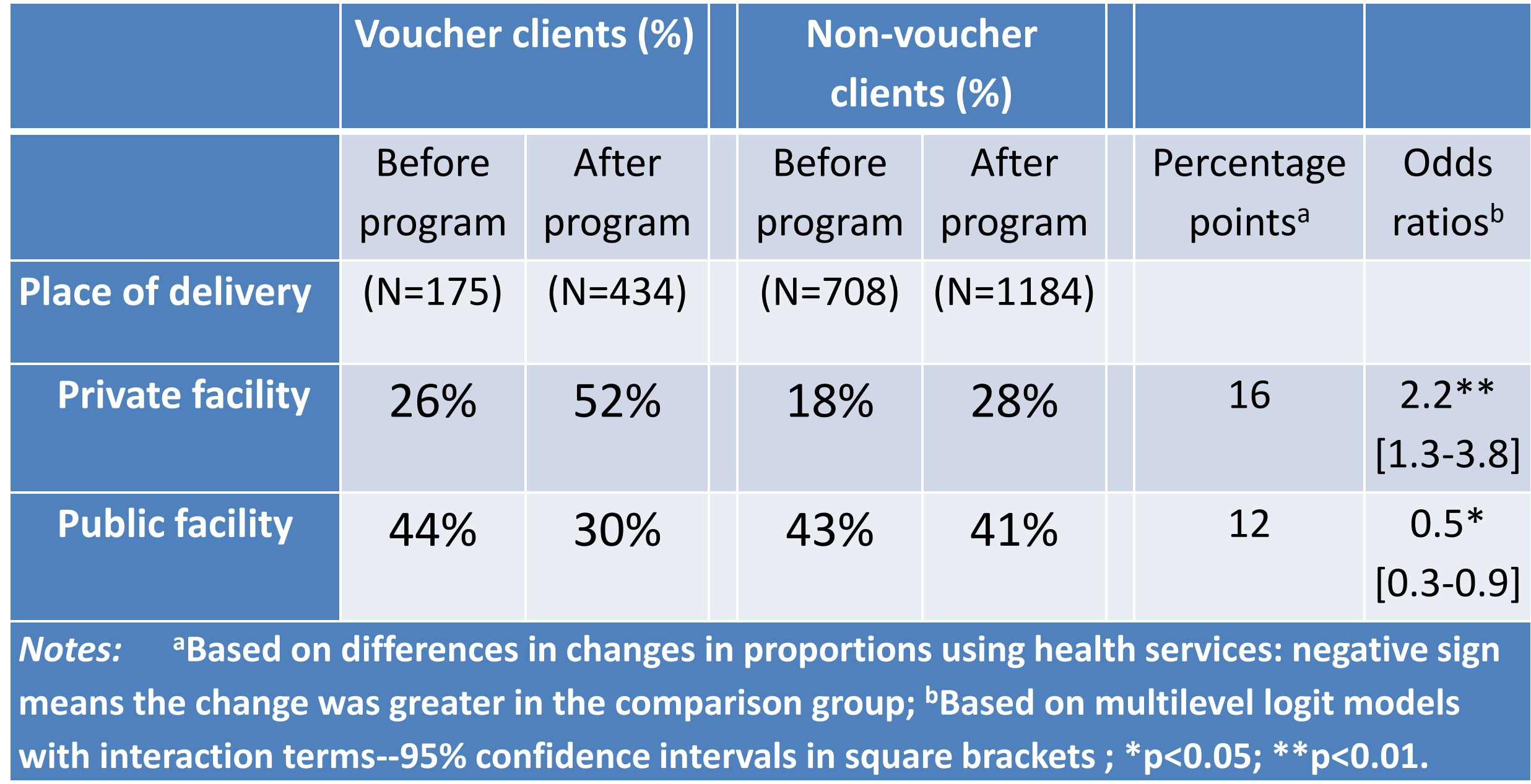

(2) Population Council 


\section{Result 3: use of ANC \& PNC}

\begin{tabular}{|c|c|c|c|c|c|c|}
\hline & \multicolumn{2}{|c|}{ Voucher clients (\%) } & \multicolumn{2}{|c|}{$\begin{array}{c}\text { Non-voucher clients } \\
(\%)\end{array}$} & \multirow[b]{2}{*}{$\begin{array}{c}\text { Percentage } \\
\text { points }^{a}\end{array}$} & \multirow[b]{2}{*}{$\begin{array}{l}\text { Odds } \\
\text { ratios }^{b}\end{array}$} \\
\hline & $\begin{array}{c}\text { Before } \\
\text { program }\end{array}$ & $\begin{array}{c}\text { After } \\
\text { program }\end{array}$ & $\begin{array}{l}\text { Before } \\
\text { program }\end{array}$ & $\begin{array}{c}\text { After } \\
\text { program }\end{array}$ & & \\
\hline $\begin{array}{l}\text { Place of } \\
\text { delivery }\end{array}$ & $(N=175)$ & $(N=434)$ & $(N=708)$ & $(N=1184)$ & & \\
\hline $\begin{array}{l}\text { Four or more } \\
\text { antenatal care } \\
\text { visits }\end{array}$ & $\begin{array}{c}55 \% \\
(N=183)\end{array}$ & $\begin{array}{c}70 \% \\
(N=459)\end{array}$ & $\begin{array}{c}49 \% \\
(N=779)\end{array}$ & $\begin{array}{c}56 \% \\
(N=1281)\end{array}$ & 8 & $\begin{array}{c}1.4 \\
{[0.9-2.2]}\end{array}$ \\
\hline $\begin{array}{l}\text { Postnatal care } \\
\text { services }\end{array}$ & $\begin{array}{c}60 \% \\
(N=183)\end{array}$ & $\begin{array}{c}67 \% \\
(N=459)\end{array}$ & $\begin{array}{c}45 \% \\
(N=779)\end{array}$ & $\begin{array}{c}53 \% \\
(N=1281)\end{array}$ & -1 & $\begin{array}{c}1.1 \\
{[0.7-1.8]}\end{array}$ \\
\hline $\begin{array}{l}\text { Notes: }{ }^{a} \text { Basec } \\
\text { means the chan } \\
\text { with interaction }\end{array}$ & n differenc & s in change & n proportic & ins using hea & services: $n$ & modive sigr \\
\hline
\end{tabular}

(1) Population Council 


\section{Result 3: Paid for most recent birth}

\begin{tabular}{|c|c|c|c|c|c|c|}
\hline & $\begin{array}{r}\text { Vouch } \\
\text { present } \\
\text { by }\end{array}$ & $\begin{array}{l}\text { client } \\
\text { n village } \\
010\end{array}$ & $\begin{array}{r}\text { No vouc } \\
\text { present } \\
\text { by }\end{array}$ & $\begin{array}{l}\text { er clients } \\
\text { in village } \\
010\end{array}$ & & \\
\hline & $\begin{array}{l}\text { Before } \\
\text { program }\end{array}$ & $\begin{array}{c}\text { After } \\
\text { program }\end{array}$ & $\begin{array}{c}\text { Before } \\
\text { program }\end{array}$ & $\begin{array}{c}\text { After } \\
\text { program }\end{array}$ & $\begin{array}{c}\text { Percentage } \\
\text { points }^{a}\end{array}$ & $\begin{array}{l}\text { Odds } \\
\text { ratios }^{b}\end{array}$ \\
\hline $\begin{array}{l}\text { Paid for last } \\
\text { delivery }\end{array}$ & & & & & & \\
\hline Private facility & $\begin{array}{c}98 \% \\
(N=206)\end{array}$ & $\begin{array}{c}54 \% \\
(N=133)\end{array}$ & $\begin{array}{c}97 \% \\
(N=112)\end{array}$ & $\begin{array}{c}86 \% \\
(N=21)\end{array}$ & 33 & $\begin{array}{c}0.1^{*} \\
{[0.0-0.9]}\end{array}$ \\
\hline $\begin{array}{l}\text { Public/private } \\
\text { facility }\end{array}$ & $\begin{array}{c}56 \% \\
(N=533)\end{array}$ & $\begin{array}{c}39 \% \\
(N=282)\end{array}$ & $\begin{array}{c}52 \% \\
(N=292)\end{array}$ & $\begin{array}{c}32 \% \\
(N=81)\end{array}$ & -3 & $\begin{array}{c}0.9 \\
{[0.4-2.1]}\end{array}$ \\
\hline
\end{tabular}

(2) Population Council 


\section{Conclusions}

- Based on household wealth index, a significantly higher proportion of women from the two poorest quintiles had used the vouchers compared to those from middle, richer and richest quintiles.

- The program significantly contributed to increased deliveries in private facilities which were accompanied by significant reductions in public facility as well as in homebased births.

- The program further significantly contributed to reductions in the likelihood of paying out-of-pocket for deliveries in private health facilities among communities exposed to it.

(Population Council 